\section{Acidente vascular cerebral precoce: implicações para adultos em idade produtiva atendidos pelo Sistema Único de Saúde}

\section{Early cerebrovascular accident: implications in working-age adults assisted by the Brazilian Public Health System}

Ilka Veras Falcão 1

Eduardo Maia Freese de Carvalho 2 Kátia Magdala Lima Barreto 3 Fabio José Delgado Lessa 4 Valéria Moura Moreira Leite 5

1,3,5 Departamento de Terapia Ocupacional. Universidade Federal de Pernambuco. Av. Prof. Moraes Rego, 1235. Cidade Universitária. Recife, PE, Brasil. CEP: 50.670-420 E mail ilkavf@nlink.com.br

2 Departamento de Estudos em Saúde Coletiva. Centro de Pesquisa Aggeu Magalhães e Departamento de Medicina Social.

Universidade Federal de Pernambuco. Recife, PE, Brasi

4 Departamento de Estudos em Saúde Coletiva. Centro de Pesquisa

Aggeu Magalhães. Recife, PE, Brasil

\begin{abstract}
Objectives: to assess incapacities and determine whether there are gender differences following an initial episode of cerebrovascular accident among survivors between the ages of 20 and 59 years old living in Recife city, hospitalized in the public health system.

Methods: in-home survey performed with a sample of survivors, investigating incapacities and alterations in the functional and productive life of these individuals.

Results: the sample had a balanced proportion between genders and an average age of 52 years old. Individuals were either illiterate or had an elementary education level, holding jobs in the informal or domestic service sector. Approximately 80\% exhibited some incapacity. Difficulty in communication and symptoms of depression were more common among women. Functional incapacities had negative repercussions in the level of fulfillment in the lives of $70 \%$ of the interviewees.

Conclusions: the percentage of cases among still young individuals that are exhibiting some type of incapacity following an early stroke episode is expressive. Strokes occur earlier and incapacities are more frequent/severe among women. Prevention and rehabilitation following a stroke are desirable with the implementation of programs that should consider gender conditions aiming at strokes' risk and incapacity control.
\end{abstract}

Key words Cerebrovascular accident, Sex, Sistema Único de Saúde.

\section{Resumo}

Objetivos: conhecer as incapacidades e identificar se há diferenças de gênero, em sobreviventes de primeiro episódio de acidente vascular cerebral (AVC), entre 20 e 59 anos de idade, na cidade do Recife e que tenham sido hospitalizados pelo Sistema Único de Saúde.

Métodos: entrevista domiciliar com uma amostra de sobreviventes, investigando-se as incapacidades referidas na vida funcional e produtiva deles.

Resultados: amostra equilibrada entre os sexos, média de idade de 52 anos, com nível elementar de estudos ou analfabeto e trabalhando no setor de serviços, informal ou doméstico. Menos de $20 \%$ informam recuperação total após o AVC. Aproximadamente $80 \%$ apresentam algum déficit, sendo os problemas de comunicação e os sintomas depressivos mais freqüentes entre as mulheres. Após o AVC aumentou o número de desempregados e aposentados e as incapacidades repercutem negativamente na satisfação de vida de mais de $70 \%$ dos entrevistados.

Conclusões: é expressivo o percentual de casos, ainda jovens, com seqüelas pós AVC, sendo este mais precoce e o quadro de incapacidades mais freqüente e/ou grave entre as mulheres. A prevenção e a reabilitação após o AVC são desejáveis, com a implantação de programas, considerando as condições de gênero, para o controle dos riscos e para as seqüelas resultantes do AVC.

Palavras-chave Acidente vascular cerebral, Sexo, Sistema Único de Saúde 


\section{Introdução}

A saúde no Brasil ainda apresenta um perfil epidemiológico marcado pela heterogeneidade. Essa heterogeneidade pode ser basicamente explicada pela distribuição desigual da riqueza, pelo inadequado acesso aos avanços científico e tecnológico, pela iniqüidade no acesso à assistência à saúde e pelas condições desiguais de desenvolvimento humano com grandes diferenças inter e intra-regionais e entre as classes sociais. 1,2

No entanto, tem sido possível observar melhorias no quadro de saúde do país com aumento da expectativa de vida ao nascer, diminuição da mortalidade materna e infantil, aumento da sobrevida e controle e erradicação de algumas doenças, principalmente, as imunopreveníveis. ${ }^{1}$ Mas, por outro lado observam-se no perfil epidemiológico atual o crescimento e precocidade de óbitos por homicídios e acidentes de trânsito na população jovem masculina e o aumento de mortes por doenças cerebrovasculares e por doença isquêmica do coração, com predominância na população feminina. Sendo ainda importante ressaltar o aumento do número de pessoas com doenças e incapacidades crônicas devido ao crescente envelhecimento populacional, o que tem gerado repercussões sociais na saúde pública e na previdência social.1,2

É nesse contexto que as doenças do aparelho circulatório adquirem relevância nos dados de morbimortalidade do país. O destaque entre essas doenças é para as cerebrovasculares e, particularmente, para o acidente vascular cerebral (AVC), que representa a terceira causa de morte em países industrializados e a primeira causa de incapacidade entre adultos. 3,4

No Brasil, a distribuição dos óbitos por doenças do aparelho circulatório vem apresentando crescente importância entre adultos jovens, já a partir dos 20 anos, assumindo o patamar de primeira causa de óbito na faixa dos 40 anos e predominando nas faixas etárias subseqüentes. A mortalidade nas capitais e em regiões metropolitanas é maior que a da população da América do Norte, onde vem declinando nos últimos 30 anos. No Recife, cidade situada em uma grande região metropolitana do Nordeste do Brasil, as doenças cardiovasculares apresentam-se como primeira causa de óbito a partir dos anos 70, com destaque, como causa de mortalidade para as doenças cerebrovasculares, o infarto agudo do miocárdio e a hipertensão arterial.5,6

O fato de as doenças do aparelho circulatório atingirem pessoas em idade produtiva tem um forte impacto econômico calculado por anos produtivos de vida perdidos, nos custos de hospitalização e de uso de serviços de saúde.1,3

Ao explorarmos o Sistema de Informação Hospitalar do Sistema Único de Saúde (SIH/SUS), no período de um ano, observamos, no total de internações de moradores do Recife, em hospitais próprios ou conveniados ao SUS no Estado, que as doenças do aparelho circulatório (DAC) ocupam o segundo lugar nas internações hospitalares, excluídas as complicações da gravidez, parto e puerpério e os transtornos mentais. No total das internações por DAC, o peso maior é o da faixa de 20 anos e mais, com, aproximadamente, $37 \%$ delas entre 20 a 59 anos, o que, possivelmente, resulta em maiores ou mais graves repercussões, por ser essa fase de intensa atividade sócio-produtiva. Por essa razão, essa faixa etária passa a ser o grupo de interesse deste estudo.

No grupo das doenças cerebrovasculares, considerando tal faixa de idade, o acidente vascular cerebral agudo (AVC) corresponde a pouco mais de 80\% das internações pelo Sistema Único de Saúde no ano investigado. Mas, além da elevada incidência, há também que se considerar as sérias conseqüências médicas e sociais que podem resultar de um AVC, como: as seqüelas de ordem física, de comunicação, funcionais, emocionais, entre outras.

Essas seqüelas implicam algum grau de dependência, principalmente no primeiro ano após o AVC, com cerca de 30 a $40 \%$ dos sobreviventes impedidos de voltarem ao trabalho e requerendo algum tipo de auxílio no desempenho de atividades cotidianas básicas.4,7 Além dos óbitos, dos custos hospitalares e previdenciários, a perda de autonomia entre adultos e a sua conseqüente dependência é uma outra forma de expressão da gravidade das incapacidades resultantes do AVC. 8

Estudos relatam serem comuns, em casos de AVC, a ansiedade, a depressão, os distúrbios do sono e da função sexual, distúrbios motores, sensoriais, cognitivos e de comunicação, e alterações fisiológicas durante atividades físicas (dispnéia, angina, hipertensão), que causam limitações para o retorno ao trabalho produtivo. 7,8

Variáveis como sexo, idade, nível educacional, severidade do AVC, vinculação a programas de reabilitação e outros fatores intrínsecos e extrínsecos à incapacidade e os seus resultados sobre o indivíduo reforçam a complexidade desses estudos, como exposto por Medina et al., 8 Angeleri et al., ${ }^{9}$ Jonkman et al., 10 e Neau et al. ${ }^{11}$

Por sua importância epidemiológica, além do potencial para a instalação de seqüelas, estudar a condição de sobreviventes de AVC residentes no Recife, revestiu-se de importância, uma vez que diag- 
nosticar e tratar o AVC agudo não significa diagnosticar e tratar as incapacidades resultantes. Segundo Stolov e Hays, 12 se o diagnóstico e o tratamento são apenas da doença, a assistência pode não ser suficiente para que o indivíduo volte a interagir normalmente com o seu meio e a incluir-se socialmente.

Considerando esses fatos, é objeto deste estudo conhecer as incapacidades e identificar se elas se diferenciam quanto ao gênero, em sobreviventes de primeiro episódio de AVC, com idade compreendida entre 20 e 59 anos, residentes no Recife, e que tenham sido hospitalizadas pelo SUS. Reforça o interesse desta investigação perceber se o AVC, doença do grupo das doenças cerebrovasculares, que são mais freqüentes no sexo feminino, têm na incapacidade também uma expressão diferenciada.

\section{Métodos}

A população foi identificada na primeira fase do estudo, entre os casos de internações, no período de um ano (2000), de sujeitos com idade entre 20 a 59 anos, na cidade do Recife, PE, Brasil, hospitalizados na rede própria ou conveniada com o SUS, tendo como diagnóstico as doenças cerebrovasculares (DCV). Para isto, exploramos a base de dados do SIH/SUS e selecionamos os casos com AVC agudo, independentemente da classificação dos subtipos.

Com esses casos construímos um banco de dados próprio e, após exclusão das informações duplicadas e dos casos de óbito hospitalar (letalidade de $32,4 \%$ ), extraímos uma listagem com 289 casos de AVC agudo, sobreviventes na ocasião da alta hospitalar, agrupados por ordem cronológica de apresentação da Autorização de Internação Hospitalar (AIH) o que nos garantiu uma posição aleatória de cada sujeito na lista, independentemente de idade, sexo ou outra condição, minimizando, assim, bias de seleção.

Para a seleção da amostra consideramos como satisfatório o número apresentado nos estudos de Rocha et al., ${ }^{7}$ Medina et al. 8 e Bonita et al., 13 os quais apontam, em média, $30 \%$ de incapacidade no primeiro ano após o AVC. Então, utilizando a técnica de amostragem sistemática e aleatória, selecionamos um em cada três casos da listagem, totalizando 96 casos.

Posteriormente, realizamos com essa amostra uma entrevista domiciliar para detectar as incapacidades, após o AVC, referidas pelos entrevistados como causadoras de modificações na sua capacidade funcional. Ou seja, as incapacidades que poderiam interferir nas habilidades físicas e mentais que de- senvolvemos ao longo da vida para uma condição independente e autônoma. 14

O instrumento de pesquisa foi um formulário estruturado, construído e testado previamente, que teve como propósito apreender a condição do entrevistado, comparativamente, antes e após o AVC. As questões foram distribuídas em seis sessões organizadas em relação: a) às informações pessoais; b) às condições de saúde anteriores e posteriores ao AVC e à instalação das incapacidades; c) à situação funcional e de desempenho das atividades de vida diária; d) ao uso do tempo livre; e) aos recursos socioeconômicos e f) à vida de relação e arranjo familiar.

Como parte dos procedimentos de pesquisa, os sujeitos e/ou familiares responsáveis foram solicitados a manifestar sua concordância com a assinatura de Termo de Consentimento, livre e esclarecido, conforme requer a Resolução 196/96 do Conselho Nacional de Saúde.

A coleta de dados ocorreu, em média, 18 meses após o AVC, estendendo-se por um período de três meses. Para a entrevista domiciliar, contou-se com três pesquisadores treinados e com os procedimentos padronizados. O teste de concordância foi realizado pelo método Kappa,15 no qual obtivemos uma ótima conformidade $(0,874)$, o que evidencia que os dados foram colhidos com confiabilidade, embora através de múltiplos pesquisadores.

Durante a realização do inquérito domiciliar, houve uma perda de sujeitos da amostra inicial em decorrência de óbito pós-hospitalar (22,9\%), de novos episódios de AVC (10,4\%) e de mudança de endereço $(15,6 \%)$. Essas causas de perda não podiam ser, a priori, controladas pelo estudo. Os casos perdidos por diagnóstico e idade diferentes dos adotados no estudo consistem em erro de informação do SIH/SUS, o que é previsível quando se trata de dados secundários, e estes foram repostos na amostra, de acordo com os procedimentos iniciais de seleção.

No entanto, esgotamos toda a listagem e não foi possível completar a amostra prevista inicialmente, pela quantidade de dados incompletos no SIH/SUS e pela grande mobilidade populacional, pois a entrevista foi realizada no domicílio dos pacientes e alguns não mais residiam no local informado à época da hospitalização. Dessa forma, a amostra final ficou constituída por 46 casos de primeiro episódio de AVC, de pacientes com idade entre 20 e 59 anos, residentes em diferentes bairros do Recife, e entrevistados, em média, 18 meses após a ocorrência.

Para registro e análise dos dados, utilizamos o programa Epi-Info, versão 6.04 . 


\section{Resultados}

\section{O perfil da amostra}

A distribuição por sexo correspondeu a $52 \%$ de homens e $48 \%$ de mulheres. Como evidenciado na Tabela 1, há maior concentração de pessoas nas faixas etárias de maior idade. A idade média foi de 52 anos, sendo de 48 anos para as mulheres e 53 anos para os homens.

O estado civil que prevalece antes e após o AVC, é casado ou união estável, e o arranjo familiar é formado por mais que duas pessoas, sem modificação por causa do AVC. Nesta população, a escolaridade predominante é de analfabetos $(39,1 \%)$ e de nível elementar com até três anos de estudo (20\%).

A profissão da população estudada centrou-se no setor de serviços. As ocupações mais freqüentes foram as de caráter manual, como pedreiro, servente, empregada doméstica, lavadeira, vigilante, etc., e somente seis casos informaram exercer uma atividade não manual, de nível médio. Em relação à profissão informada, a quase totalidade das mulheres se ocupava de atividades vinculadas ao espaço doméstico (lavadeira, cozinheira, empregada doméstica), sendo que, aproximadamente, 1/3 destas eram "donas de casa" e não possuíam vínculo empregatício.

Quanto à situação profissional, foi observado que o AVC trouxe modificações, com redução da condição de trabalhadores. Antes do AVC, 83\% dos homens e $54 \%$ das mulheres encontravam-se trabalhando; após o AVC, apenas $25 \%$ dos homens e $4,5 \%$ das mulheres mantiveram essa condição.

Após o AVC, a fonte de renda principal deixou de ser o trabalho (assalariado, autônomo ou informal) sendo substituída pela aposentadoria e por benefício previdenciário, para quase $50 \%$ dos homens e $32 \%$ das mulheres. Aproximadamente, $10 \%$ dos homens e $45 \%$ das mulheres passaram a ter como fonte de renda as doações ou recursos de familiares.

\section{O quadro de incapacidades instaladas após o AVC}

Menos de 20\% referiram recuperação total. $\mathrm{Na}$ Tabela 2, são apresentados, as incapacidades ou problemas resultantes do AVC, como referidos pelos entrevistados. A incapacidade motora foi atribuída basicamente à redução dos movimentos do hemicorpo a seguir da perna e, em menor proporção, por redução dos movimentos do braço. A mobilidade ou condição de andar em casa e no seu entorno se encontrava preservada em aproximadamente, $90 \%$ dos casos. No entanto, pouco mais da metade das pessoas tinha impedimento para deslocar-se para outros bairros e entre as que se deslocavam havia necessidade da ajuda familiar e/ou do uso de dispositivos auxiliares, como bengala, cadeira de rodas, andador, entre outros.

\section{Tabela 1}

Distribuição por grupo etário nos casos de primeiro episódio de acidente vascular cerebral (AVC) na cidade do Recife, hospitalizados pelo Sistema Único de Saúde (SUS).

\begin{tabular}{lcr}
\hline Grupo etário & $\mathbf{n}$ & $\%$ \\
\hline 20 a 29 anos & 3 & 6,6 \\
30 a 39 anos & 4 & 8,7 \\
40 a 49 anos & 13 & 28,2 \\
50 a 59 anos & 26 & 56,5 \\
Total & 46 & 100,0 \\
\hline
\end{tabular}

\section{Tabela 2}

Distribuição das incapacidades informadas pelos sobreviventes de acidente vascular cerebral (AVC), hospitalizados pelo Sistema Único de Saúde (SUS) na cidade do Recife, durante o inquérito domiciliar.

\begin{tabular}{lcc}
\hline Incapacidade/Seqüelas & Homem (\%) & Mulher (\%) \\
\hline Motora (movimentos) & 75,0 & 90,9 \\
Mobilidade em casa & 8,4 & - \\
Mobilidade no entorno de casa & 16,7 & 9,1 \\
Mobilidade em outros bairros (na cidade) & 45,9 & 54,5 \\
De comunicação (falar/entender) & 50,0 & 68,1 \\
De audição & 20,8 & 31,8 \\
De visão & 50,0 & 54,5 \\
Sintomas de depressão & 50,0 & 90,9 \\
Memória & 41,6 & 59,0
\end{tabular}


Uma outra incapacidade referida foi a de comunicação, sendo a afasia (de compreensão ou motora) um problema importante que ocorreu em $50 \%$ dos homens e em quase $2 / 3$ das mulheres. Foram referidas dificuldades de articulação das palavras e lentidão para falar interferindo nas relações interpessoais.

Todos esses problemas de comunicação foram relacionados à depressão. A referência a sintomas de depressão foi particularmente expressiva entre as mulheres, mas também para a metade dos homens. Todos os casos entrevistados apontaram a mudança da capacidade funcional interferindo na vida pessoal, modificando sua autonomia e auto-estima. A condição de vida atual é insatisfatória para $77,2 \%$ das mulheres e $70,8 \%$ dos homens, tendo como motivos principais as incapacidades, a situação financeira e profissional e as preocupações com a sobrevivência pessoal e familiar.

O conhecimento de doença pré-existente associada ao AVC, entre as mulheres, é de quase $73 \%$ e entre os homens de menos de $42 \%$, sendo a hipertensão arterial a doença mais referida. Dentre os que conheciam uma doença anterior ao AVC, 50\% das mulheres realizaram tratamento contra $20 \%$ dos homens. No momento da entrevista, cerca de $30 \%$ dos casos não estavam em tratamento médico-medicamentoso e entre os demais foi freqüente a irregularidade do tratamento e a falta de acompanhamento por profissional de saúde.

\section{Discussão}

Esta pesquisa referiu-se a adultos em idade produtiva, com proporção equilibrada entre os gêneros. A idade média de 52 anos e a concentração dos casos nas faixas etárias de 40 a 59 anos representam a tendência de maior ocorrência de AVC em pessoas de mais idade, como citado em outros estudos.4,13,16 A ocorrência do primeiro episódio de AVC, observada em Joinville, 16 foi de 65,2 anos e no estudo de Framingham, conforme Jette et al., 17 é de 64 anos. Portanto em nosso meio, há uma certa tendência à precocidade do primeiro episódio de AVC.

A profissão da maioria dos entrevistados, em atividades de pouca qualificação profissional ("trabalho braçal" ou de menor envolvimento intelectual), no setor de serviços, de atividade informal ou doméstico, especialmente, em relação às mulheres, pode refletir uma escolaridade precária. Isso se constitui em ponto negativo para a recuperação, pois evidencias da literatura, relacionam escolaridade mais elevada com aumento da sobrevida, melhor controle de fatores de riscos para doenças cardiovasculares e melhor capacidade de retornar ao trabalho. $8,18,19$

Essa condição de trabalho parece refletir um papel sociocultural, aprendido e bem delimitado, do feminino em oposição ao masculino. À mulher se reserva o "mundo da casa" (espaço privado), as tarefas de servir e atender aos outros e ao homem se destina o "mundo da rua" (espaço público), com atividades independentes e complexas. ${ }^{20}$ Nesse caso, mesmo as mulheres que trabalham no espaço público, são em tarefas tidas como "próprias da mulher": cuidar da casa, da roupa, das crianças, da alimentação, ou em profissões reconhecidas como femininas, como secretária e auxiliar de enfermagem.

Quanto ao status profissional, esse foi alterado com o AVC, deslocando-se da condição de trabalhador para a de aposentado. As mulheres encontram-se, após o AVC, em uma situação mais precária, porque a fonte de renda principal passa a ser a de terceiros, especialmente porque o trabalho anterior era informal e sem cobertura previdenciária. A manutenção da condição de trabalho após o AVC para uma população em idade produtiva é inferior ao encontrado na literatura. Neau et al. 11 relatam que em uma população de 15 a 45 anos o retorno ao trabalho de pouco mais de $70 \%$, em média, após oito meses do AVC, embora tenha havido necessidade de ajustes na ocupação de, cerca de $26 \%$. Ching-Lin e Mong-Hong 19 encontraram $60 \%$ de retorno ao trabalho, com volta completa de quase metade destes e limitação na jornada ou tipo de trabalho do restante. O presente estudo relata também uma associação entre retorno ao trabalho a um melhor nível de educação e instituição de emprego.

Esses estudos associam alguns fatores que influenciam na condição de retorno ao trabalho, destacando como sendo negativos a depressão, a afasia, o local de trabalho sem possibilidade de ajustes de função, a gravidade do quadro motor e a limitação da mobilidade. Como fatores positivos consideram: terapia de reabilitação, habilidade para a fala, maior nível de educação e condições da instituição de emprego, (por exemplo, a estabilidade existente para os funcionários públicos).

Fica evidente portanto, a desvantagem dos casos aqui estudados, pois muitos indivíduos são analfabetos ou de baixa escolarização ocupando vagas no setor informal, o que é indicativo de situação de pobreza e de baixas oportunidades anteriores ao AVC. Tal situação pode ser agravada, evidentemente, pelas incapacidades residuais dele resultantes.

A recuperação total (considerada como retomada do trabalho) em torno de $20 \%$, após o AVC, encon- 
trada em nosso estudo aproxima-se de alguns dados da literatura. A esse respeito, Reddy e Reddy 21 estimam em $10 \%$ os sobreviventes sem seqüelas; Bonita et al., 13 apresentam taxas de funcionamento independente, após o AVC, em torno de $25 \%$. Por outro lado Neau et al., 11 em uma população jovem, encontraram $2 / 3$ de sobreviventes sem nenhum problema. Uma recuperação completa está associada a vários fatores, como a área cerebral atingida, a idade, o tempo entre os sinais do AVC e o atendimento, entre outros. Esses autores, ressaltam que há uma interrelação de vários fatores, de difícil controle, para determinar o que leva alguns casos a uma total recuperação e outros à severa dependência. Em nosso estudo, não associamos à recuperação fatores que possam explicá-la.

Nosso achado em relação à incapacidade motora é elevado, se comparado à condição de mobilidade no interior do domicílio, quando verificamos que, menos de, $10 \%$ dos casos têm essa limitação. Por se tratar de uma população relativamente jovem, a condição de mobilidade dos casos estudados é melhor e difere do observado por Lincoln et al.22 que encontraram um índice de $27 \%$ com problemas de mobilidade severos entre paciente idosos. Entretanto, verificamos que em nosso meio os problemas de mobilidade se ampliam quando considerados o entorno do domicílio e a mobilidade para outros bairros da cidade.

Quanto às incapacidades sensoriais, os problemas visuais, de acordo com os entrevistados, estão relacionados à redução da habilidade para as atividades de vida diária, como vestir-se, cuidar da aparência e realizar atividades de lazer, tais como: ler e assistir à televisão. Em relação à perda auditiva, o maior impacto é no convívio e nas relações interpessoais. Nossos achados são compatíveis com a literatura 23,24 que refere correlação entre limitações de visão e audição e deterioração no estado funcional e no convívio social.

Quanto à depressão em sobreviventes de AVC, vários estudos informam que é freqüente os pacientes não serem diagnosticados e tratados.7,10,12,25 Para Rocha et al.,7 é importante diferenciar os sintomas depressivos e a depressão clínica: a tristeza que se manifesta precocemente após o quadro do AVC, como reação ao adoecimento e suas consequiências, o que pode ser considerado natural, e o quadro de sintomatologia mais severa, com alterações do sono, do humor, perda de interesse, de concentração, entre outras alterações. Mas, independentemente da severidade, o diagnóstico e o tratamento do quadro depressivo são desejáveis porque influem positivamente na recuperação como um todo.

Os estudos diferem em relação à incidência de depressão após o AVC, com taxas de 20 a $60 \%$, no estudo de Rocha et al., 7 e 48,31\% no estudo de Neau et al. ${ }^{11} \mathrm{O}$ estudo de Rocha et al. (1993: 204)7 também revela que "... uma maior incidência e severidade dos quadros depressivos entre o $6^{\circ}$ e $24^{\circ}$ mês após o AVC pode sugerir a participação de fatores psicológicos de ajustamentos tardios." A ocasião em que entrevistamos a população, em média 18 meses após o AVC, corresponde a esse período, o que poderia justificar a ocorrência freqüiente de sintomas depressivos em nossa amostra com valor muito elevado, especialmente, entre as mulheres (90\%).

Ainda em relação à depressão, há relatos de uma associação entre sexo feminino e idade, ${ }^{14,26}$ o que não foi possível confirmar no presente estudo, pois a quase totalidade das mulheres entrevistadas, independentemente da faixa etária, informa sintomas depressivos por ocasião do inquérito domiciliar.

Em relação ao sexo, observamos que todas as mulheres, com exceção de uma, que revelam problemas de comunicação, informam depressão, e entre os homens essa relação é encontrada em $2 / 3$. Tal associação parece-nos relevante e condiz com os estudos citados, levantando uma questão complexa de ser esclarecida: a depressão é induzida por dificuldades de comunicação ou o inverso? Parece-nos razoável que, como as dificuldades de comunicação são posteriores ao evento (AVC) essas influenciem a ocorrência da depressão.

Das incapacidades estudadas, de modo geral, os valores são maiores em relação às mulheres, o que pode indicar que as incapacidades são mais freqüentes ou mais graves entre elas. No entanto, esse achado pode estar influenciado por um viés de gênero, uma vez que homens e mulheres assumem comportamentos diferentes em relação aos seus problemas de saúde. 27

Estudos mostram a mulher mais atenta, com facilidade maior em falar e reconhecer os problemas de saúde e seus sintomas e em procurar e usar os serviços preventivos e para tratamento, o que estaria atrelado à "condição feminina" associada à maternidade e à responsabilidade pela conservação da vida, fazendo com que as mulheres tenham uma ligação especial com a saúde. Neste estudo quando comparamos homens e mulheres quanto ao conhecimento e tratamento de doença hipertensiva, pré-existente ao AVC, os valores positivos são maiores entre as mulheres.

A literatura associa fatores de risco às doenças cerebrovasculares e, invariavelmente, destaca a hipertensão arterial como um dos mais importantes e mais fortemente correlacionados ao AVC, como também ressalta que a terapia anti-hipertensiva é capaz 
de reduzir a morbidade e mortalidade por essa causa. 5,28-30 No entanto, concordamos com Hale $e t$ $a l .,{ }^{28}$ quando afirmam que o fato de ter conhecimento de ser hipertenso e receber medicação não é o bastante para prevenir suas conseqüências, uma vez que, de acordo com os autores, poucos pacientes (apenas $20 \%$ ) associavam hipertensão e AVC, demonstrando um conhecimento menor que o desejável.

Em nosso meio, constatamos que, mesmo sabendo-se portador de doença hipertensiva e ainda após a primeira hospitalização por AVC, muitos pacientes continuavam sem um controle adequado da hipertensão arterial. Fato que pode determinar a ocorrência de novos episódios de AVC, de aumento do potencial de instalação de incapacidades, além do aumento da letalidade.

\section{Conclusões}

Neste estudo, evidenciamos como sendo relevante a distribuição do AVC em adultos com idade entre 20 e 59 anos e o expressivo percentual de casos, cerca de $80 \%$, após o primeiro episódio de AVC, já referindo algum tipo de incapacidade, o que pode ser especialmente negativo para uma população em idade economicamente ativa.

Também verificamos que as incapacidades, após o AVC, repercutem ou são reveladas de modo diferente para homens e mulheres e que os sintomas depressivos e problemas de comunicação apresentam maior força entre as mulheres. Mas, o AVC, independentemente do gênero, é causa de insatisfação com a vida e de limitações funcionais diversas, pela perda da autonomia decorrente das incapacidades.

Assim, o setor saúde precisa organizar-se para lidar com essa população incapacitada como também para implementar ações visando ao controle efetivo dos fatores de risco do AVC. Não somente com a terapia anti-hipertensiva clássica, mas que a esta sejam associados programas de adesão ao tratamento, com conscientização da prevenção e orientação para os riscos e às conseqüências correlacionadas ao AVC e às suas seqüelas. É ainda desejável que sejam consideradas, ao se planejarem tais ações, a condição de gênero e os comportamentos preventivos e de cuidados com a saúde, diferentes, assumidos por sexo.

\section{Referências}

1. OPAS (Organização Panamericana da Saúde), OMS (Organização Mundial da Saúde). A saúde no Brasil. Brasília (DF): As Organizações; 1998.

2. Carvalho EF, Lessa F, Gonçalves FR, Silva JAM, Lima MEFL, Melo Júnior SW. O processo de transição epidemiológica e iniqüidade social: o caso de Pernambuco. Rev Assoc Saúde Pública Piauí 1998; 1: 107-19.

3. Kaste M, Fogelholm R, Rissanen A. Economic burden of stroke and the evaluation of news therapies. Public Health 1998; 112: 103-12.

4. Pereira CF, Lemos MM, Benevenuto MC, Fonseca GA. Enfoque sobre pesquisa prospectiva no AVC. Med Reabil 1993; (34/36): 9-13.

5. Carvalho EFM. Estúdio de los fatores psicosociales asociados com la hipertension arterial primaria [tese doutorado]. Madrid: Faculdad de Medicina, Universidad Complutense; 1993.

6. Chor D, Fonseca MJM, Andrade CR, Waissmann W, Lotufo PA. Doenças cardiovasculares: panorama da mortalidade no Brasil. In: Minayo MCS, organizadora. Os muitos Brasis: saúde e população na década de 80. São Paulo: Hucitec; 1995. p. 57-86

7. Rocha FL, Cunha UGV, Giiacomin KC. Depressão pós-aci- dente vascular cerebral (AVC). J Bras Psiquiatr 1993; 42: 203-8.

8. Medina MCG, Shirassu MM, Goldefer MC. Das incapacidades e do acidente cerebrovascular. In: Karsch UMS, organizadora. Envelhecimento com dependência: revelando cuidadores. São Paulo: EDUC; 1998. p. 199-214.

9. Angeleri F, Angeleri VA, Foschi N, Ciaquinto S, Nolfe G. The influence of depression, social activity, and family stress on functional outcome after stroke. Stroke 1993; 24: 478-83.

10. Jonkman EJ, De Weerd AJ, Vrijens NL. Quality of life after a first ischemic stroke. Long-term developments and correlations with changes in neurological deficit, mood and cognitive impairment. Acta Neurol Scand 1998; 98: 16975.

11. Neau JP, Ingrand P, Mouille-Brachet C, Rosier MP, Couderq C, Alvarez UM, Gil R. Functional recovery and social outcome after cerebral infarction in young adults. Cerebrovasc Dis 1998; 8: 296-302.

12. Stolov WC, Hays RM. Avaliação do paciente. In: Kottke FJ, Lehmann JF, editores. Krusen, Tratado de medicina física e reabilitação. 4. ed. São Paulo: Manole; 1994. p. $1-18$ 
13. Bonita R, Solomon N, Broad J. Prevalence of stroke-related disability: estimates from the Auckland stroke studies. Stroke 1997; 28: 1898-902

14. Vieira EB. Manual de gerontologia: um guia teórico prático para profissionais, cuidadores e familiares. Rio de Janeiro: Revinter; 1996.

15. Andrade ALSS, Zicker F. Métodos de investigação epidemiológica em doenças transmissíveis. Brasília (DF): OPAS; 1987.

16. Cabral NL, Longo AL, Moro CHC, Amaral CH, Kiss HC. Epidemiologia dos acidentes cerebrovasculares em Joinville, Brasil: estudo institucional. Arq Neuropsiquiatr 1997; 55: 357-63.

17. Jette AM. Pinsky JL, Branch LG, Wolf PA, Feinelib M. The Framinghan disability study: physical disability among community-Dwelling survivors of stroke. J Clin Epidemiol 1988; 41: 719-26.

18. Santana BS, Fukujima MM, Oliveira RMC. Características socioeconômicas de pacientes com acidente vascular cerebral. Arq Neuropsiaquiatr 1996; 54: 428-32.

19. Ching-Lin H, Mong-Hong L. Factors influencing vocational autcomes following stroke in Taiwan: a medical centre-based study. Scand J Rehabil Med 1997; (29): 113 20 .

20. Santi PH. Rol de gênero y funcionamiento familiar. Rev Cubana Med Gen Integr 2000; 16: 568-73.

21. Reddy PM, Reddy V. Stroke rehabilitation. Am Fam Physician 1997; 55: 1742-8

22. Lincoln NB, Parry RH, Vass CD. Randomizes, controlled trial to evaluate increased intensity of physioterapy treat- ment of arm function after stroke. Stroke 1999; 30: 5739.

23. Harvey RL, Roth EJ, Heinemann AW, Lovell LL, McGuire JR, Diaz S. Stroke rehabilitation: clinical predictors of resource utilization. Arch Physioter Med Rahabil 1998; 79: 1349-55.

24. Rubio KB, Van Deusen J. Relation of perceptual and body image dysfuntion to activities of daily living of persons after stroke. Am J Occup Ther 1995; 49: 551-9.

25. Harwood RH, Gompertz P, Ebrahim S. Handicap one year after a stroke: validity of a new scale. J Neurol Neurosurg Psychiatr 1994; 57: 825-9.

26. Wilkinson PR, Wolf CD, Warburton FG, Rudd AG, Howard RS, Ross-Russel RW, Beech RR. A long-term follow-up of stroke patients. Stroke 1997; 28: 507-12.

27. Travassos C, Viacava F, Pinheiro R, Brito A. Utilização dos serviços de saúde no Brasil: gênero, características familiares e condição social. Rev Panam Salud Publica 2002: 11: 365-73.

28. Hale LA, Fritz VU, Eales CJ. Do stroke pacients realise that a consequence of hypertension is stroke? S Afr Med J 1998; 88: 451-4.

29. Zétola VHF, Nóvak EM, Camargo CHF, Carraro Júnior H, Coral P, Muzzio JA, Iwamoto FM, Coleta MVD, Werneck LC. Acidente vascular cerebral em pacientes jovens: análise de 164 casos. Arq Neuropsiquiatr 2001; 59: 740-5.

30. Ortiz GF. Prevalencias, discapacidades, fallecimientos y costos de la enfermedad vasculocerebral en México. Arch Neuroci Mex 2000; 5: 205-10.

Recebido em 8 de abril de 2002

Versão final reapresentada em 22 de setembro de 2003

Aprovado em 25 de novembro de 2003 